\title{
Frequency modulation technique for wide-field imaging of magnetic field with nitrogen-vacancy ensembles
}

Yukihiro Miura ${ }^{1}$, Satoshi Kashiwaya ${ }^{2}$, and Shintaro Nomura ${ }^{1 *}$

${ }^{1}$ Division of Physics, Univ. of Tsukuba, Tennoudai, Tsukuba, Ibaraki 305-8571, Japan

${ }^{2}$ National Institute of Advanced Industrial Science and Technology, Umezono, Tsukuba, Ibaraki 305-8568, Japan

*E-mail: nomura.shintaro.ge@u.tsukuba.ac.jp

We report on the application of a frequency modulation technique to wide-field magnetic field imaging of nitrogen-vacancy centers in diamond at room temperature. We use a scientific CMOS (sCMOS) camera to collect photoluminescence images from a large number of nitrogen-vacancy center ensembles in parallel. This technique allows a significant reduction in measurement time to obtain a magnetic field image as compared with a scanning probe approach at a comparable magnetic field sensitivity. 


\section{Introduction}

The unique electronic properties of negatively charged nitrogen-vacancy (NV) centers in diamond enable the measurement of magnetic field by optically detected magnetic resonance. Magnetometry utilized on NV centers is a promising approach for solid-state sensors operated at room temperature. ${ }^{1-5}$ The direct measurement of magnetic fields in nanoscale devices and materials has recently been intensively investigated using various solid-state devices such as superconducting quantum interference devices (SQUIDs), ${ }^{6-12}$ Hall sensors, ${ }^{13-16}$ and magnetic force microscopes (MFMs). ${ }^{17-19}$ Among these, SQUIDs are considered as the most highly magnetic field sensitive probes to date. Recently, highresolution imagings have been performed using SQUIDs, ${ }^{9-12}$ but magnetic field sensitivity tends to be degraded with a reduction in the size of the SQUID loop. In particular, a long measurement time is typically required in these devices because the scanning probe method is utilized to obtain images and the magnetic field is measured sequentially point-by-point.

High sensitivity to a magnetic field has been demonstrated by using the quantum coherence of the spins of highly localized electrons in NV centers. Magnetometry using NV centers is a promising technique for achieving both high spatial resolution and high sensitivity. Sensitivity to a dc magnetic field is limited by the linewidth of ESR proportional to $1 / T_{2}{ }^{*}$, where $T_{2}{ }^{*}$ is the inhomogeneous dephasing time. ${ }^{1-5}$ The magnetic field sensitivity of a wide-field magnetic field microscope using NV ensembles is degraded from a scanning-probe microscope using a single NV center because $T_{2}{ }^{*}$ of the electrons in $\mathrm{NV}$ ensembles is shorter than $T_{2}{ }^{*}$ of the electrons in a single NV center. ${ }^{3}$ However, by accumulating photoluminescence (PL) in parallel using multiple pixels of a camera, ${ }^{20-23}$ the total measurement time can be reduced at a comparable magnetic field sensitivity. This parallel acquisition of a magnetic field image is one of the advantages of NV-center-based magnetic field microscopy over other methods such as those using SQUIDs, Hall probes, and MFMs. Recently, a rectangular frequency modulation of the microwave has been employed for highly sensitive magnetometry. ${ }^{24}$ This method has been demonstrated to have a comparable or slightly improved magnetic field sensitivity under an optimum condition compared with the Ramsey fringe method. ${ }^{3}$ This frequency modulation method has the advantages of fast response in magnetic field measurement and in requiring less demanding resources compared with the method using a Ramsey-type pulse sequence. Part of this paper was previously published in an extended abstract, ${ }^{25}$ in which we 
descrubed an optically detected magnetic resonance spectrum by the frequency modulation of a (100) type IIa CVD diamond chip with native ${ }^{14} \mathrm{~N}$ and ${ }^{15} \mathrm{~N}$ impurities. In this study, we report on the application of the frequency modulation method to a wide-field magnetic field imaging of nitrogen-vacancy centers in a ${ }^{15} \mathrm{~N}_{2}{ }^{+}$implanted diamond chip at room temperature. We demonstrate that the total measurement time at a comparable sensitivity is shorter in the wide-field magnetic field imaging than in the scanning probe method owing to the parallel acquisition of magnetic field images by a scientific CMOS (sCMOS) camera.

\section{Experimental methods}

We used a (100)-oriented CVD-grown high purity single crystal diamond chip with dimensions of $2.0 \times 2.0 \times 0.5 \mathrm{~mm}^{3}$ (Element 6). N concentration was less than $5 \mathrm{ppb} .{ }^{15} \mathrm{~N}_{2}{ }^{+}$ ions were implanted ${ }^{26-28}$ at $10 \mathrm{keV}$ at a fluence of $1 \times 10^{13} \mathrm{~cm}^{-2}$. The diamond chips were annealed at $800{ }^{\circ} \mathrm{C}$ for $30 \mathrm{~min}$ and cleaned by acid. A schematic diagram of the measurement setup for wide-field imaging is shown in Fig. 1. A single turn coil with the diameter of $1 \mathrm{~mm}$ and a width of $50 \mu \mathrm{m}$ was prepared on a sapphire substrate by photolithography and was placed on the diamond chip. The single turn coil was used to apply the microwave to NV centers in diamond with a microwave signal generator at the output power of $17 \mathrm{dBm}$. A temperature- and power-stabilized semiconductor laser diode was used for optical excitation at the wavelength of $520 \mathrm{~nm}$. A microscope objective 100x, NA 0.73 (Nikon, CF IC EPI Plan SLWD) with a working distance of $4.7 \mathrm{~mm}$ was used to illuminate the diamond chip and collect PL. The excitation laser light was focused to the back aperture of the microscope objective. The laser power at the incident of the microscope objective was $6.5 \mathrm{~mW}$. The size of the laser beam spot on the diamond chip was approximately $10 \times 30 \mu \mathrm{m}^{2}$. The image of the PL from negatively charged NV centers was acquired by a cooled sCMOS camera after passing through a long-wavelength pass optical filter with a cut-on wavelength of $650 \mathrm{~nm}$. An external magnetic field was applied by $\mathrm{Nd}_{2} \mathrm{Fe}_{14} \mathrm{~B}$ permanent magnets on two-axis rotation stages to vary the direction of the magnetic field.

\section{Results}

Figure 2(a) shows an optically detected magnetic resonance spectrum of NV ensembles at room temperature for the external magnetic field $\mathbf{B} / /$ [001] as schematically shown in Fig. 2(b). The PL intensity under microwave irradiation was normalized by the PL 
intensity in the absence of microwave irradiation. The exposure time of each frame was 20 ms and a total of $100 \times 2$ frames were captured at each microwave frequency with and without microwave irradiation. The Hamiltonian of an $|\mathbf{S}|=1$ electron in NV centers is given by $^{29}$

$$
\begin{aligned}
H= & h D S_{z}^{2}+g \mu_{B} B_{N V} S_{z}+g \mu_{B}\left(B_{x} S_{x}+B_{y} S_{y}\right)+h E\left(S_{x}^{2}-S^{2}{ }_{y}\right) \\
& +A_{g S}^{\|} S_{z} I_{z}+A^{\perp}{ }_{g S}\left[S_{x} I_{x}+S_{y} I_{y}\right]+\mu_{N} g_{N} \mathbf{I} \cdot \mathbf{B},
\end{aligned}
$$

where $D, \mathbf{B}, E, g$, and $\mu_{\mathrm{B}}$ are the zero field splitting energy of $2.87 \mathrm{GHz}$ at room temperature, the external magnetic field, the splitting energy by lattice deformation, the gyromagnetic factor, and the Bohr magneton, respectively, Moreover, $A_{g s}^{\|}$and $A_{g s}^{\perp}$ are the axial and non-axial magnetic hyperfine parameters, respectively, $\mu_{\mathrm{N}}$ is the nuclear magneton, $g_{\mathrm{N}}$ is the isotropic nuclear $g$-factor of ${ }^{15} \mathrm{~N}$, and $\mathbf{I}$ is the nuclear spin of ${ }^{15} \mathrm{~N}$ [Ref. 29].

For the case of $D>>B>h E /\left(g \mu_{B}\right)$, the resonance frequencies $v_{ \pm}$are given by ${ }^{1,29}$

$$
v_{ \pm}\left(B_{N V}\right)=D \pm \frac{g \mu_{B}}{h} B_{N V}
$$

where $B_{\mathrm{NV}}$ is the projection of the external magnetic field $\mathbf{B}$ to the axis of an NV center, $\mathrm{NV} i$, for $i=1,4$. This enables the measurement of a local magnetic field vector. ${ }^{30}$ In the case of the $\mathbf{B} / /[001]$ direction, the projections of $\mathbf{B}$ to the four directions of $\mathrm{NV}_{1}-\mathrm{NV}_{4}$, i.e., [1-1-1], [111], [-11-1], and [-1-11] directions, are equivalent. As a result, two resonance dips are observed in Fig. 2(a). By contrast, four resonance dips are observed in Fig. 2(c) in the case of the $\mathbf{B} / /[111]$ direction because we have $B_{\mathrm{NV} 2}=B$ and $B_{\mathrm{NV} 1}=B_{\mathrm{NV} 3}=B_{\mathrm{NV} 4}=B / \sqrt{ } 3$ in this case. The FWHM of the dips at 2.745 and $2.996 \mathrm{GHz}$ was $8 \mathrm{MHz}$, corresponding to the resonance in the [111] direction, while the FWHM of the peaks at 2.838 and 2.919 $\mathrm{GHz}$ was $14 \mathrm{MHz}$, corresponding to the resonances in the [1-1-1], [-11-1], and [-1-11] directions. The FWHM of the peaks at 2.838 and $2.919 \mathrm{GHz}$ was broadened by the slight misalignment of the direction of the magnetic field.

Magnetic field sensitivity is defined as ${ }^{24}$

$$
\eta=\frac{\sigma}{d I_{\mathrm{PL}} / d \nu} \frac{1}{\gamma},
$$

where $I_{\mathrm{PL}}$ and $\sigma$ are the normalized PL intensity and the standard deviation of the PL intensity, respectively, and $\gamma$ is the gyromagnetic factor $\gamma=28 \mathrm{kHz} / \mu \mathrm{T}$. We obtained $\eta=47 \mu \mathrm{T} /(0.65 \mu \mathrm{m})^{2} / \sqrt{\mathrm{Hz}}$ at around $v=3.000 \mathrm{GHz}$ in the case of $\mathrm{B} / /[111]$ in Fig. 
2(c) by binning 10x10 pixels of the sCMOS camera.

An optically detected magnetic resonance spectrum by frequency modulation is shown in Fig. 3 as a function of the microwave center frequency $f_{0}$ for the external magnetic field $\mathbf{B} / /[111]$. The microwave frequency is modulated $^{24}$ as given by

$$
f(t)=f_{0}+f_{\bmod } \Sigma\left[\cos \left(2 \pi r_{\text {mod }} t\right)\right],
$$

where $f_{\text {mod }}$ and $r_{\text {mod }}$ are the modulation amplitude, and the modulation rate, respectively, and $\Sigma(t)= \pm 1$ is the sign function. The PL images were obtained by the exposure time of 20 ms at $f_{0}+f_{\bmod }$ and by another exposure time of $20 \mathrm{~ms}$ at $f_{0}-f_{\bmod }$ at $f_{\text {mod }}=1 \mathrm{MHz}$ and $r_{\text {mod }} \sim 20 \mathrm{~Hz}$. This procedure was repeated for 100 cycles at each microwave center frequency. The obtained optically detected magnetic resonance spectrum in Fig. 3(a) is proportional to the first derivative of the spectrum in Fig. 2(c) with respect to v. Figure 3(b) shows a magnified view of the plot in Fig. 3(a) that clearly shows the splliting of $\sim 3 \mathrm{MHz}$ in the optically detected magnetic resonance spectrum due to the hyperfine interaction in agreement with the previous results. ${ }^{26,31}$ As a result of frequency modulation, $d I_{\mathrm{PL}} / d v$ is larger in Fig. 3(a) than in Fig. 2(c). We obtained a magnetic field sensitivity of $\eta=21 \mu \mathrm{T} /(0.65 \mu \mathrm{m})^{2} / \sqrt{\mathrm{Hz}}$ at around $2.747 \mathrm{GHz}$.

\section{Discussions}

The magnetic field sensitivity of $\eta_{\mathrm{s}}=6 \mu \mathrm{T} / \sqrt{\mathrm{Hz}}$ was achieved by measurements with the frequency modulation of microwaves in scanning probe approach with a single-channel detector. ${ }^{24}$ Magnetic field sensitivity in the scanning probe approach has the advantages of a higher modulation rate of $\sim 100 \mathrm{kHz}$, but the total measurement time to acquire an image is $N_{\text {pixel }}$ times the measurement time of a single spot. Althpugh the magnetic field sensitivity $\eta_{\mathrm{m}}$ in the wide-field multichannel detection method is degraded from the optimum case of single-channel detection $\eta_{\mathrm{s}}$, the total measurement time at a fixed sensitivity is shorter if $\sqrt{N_{\text {pixel }}}$ exceeds $\eta_{\mathrm{m}} / \eta_{\mathrm{s}}$. In fact, a PL image of 528 x 512 pixels in a field of view of $35 \times 34 \mu \mathrm{m}$ was acquired in parallel in our wide-field imaging method within $12 \mathrm{~s}$ as compared with $\sim 10 \mathrm{~min}$ in the scanning probe approach at a comparable magnetic field sensitivity. ${ }^{24}$

The optimal modulation rate that gives the highest magnetic field sensitivity is given by $^{24}$ 


$$
f_{\text {mod }}=\sqrt{\frac{2}{\ln 2}} \frac{1}{T_{2}^{*}} .
$$

Currently, the modulation rate is limited by the frame rate of the sCMOS camera of $419 \mathrm{~Hz}$ at $528 \times 512$ pixels. The main source of the noise was read noise of $1.45 \mathrm{e}^{-}$. This may be effectively reduced by using a camera with a larger pixel size of, for example, $65 \times 65 \mu$ $\mathrm{m}^{2}$, thereby increasing the number of photoelectrons per pixel while keeping the spatial resolution of $0.65 \mu \mathrm{m}$ limited by the pixel size. The optimum microwave and laser power decrease with an increase in the rate of photoelectrons per pixel ${ }^{32}$. As a result, magnetic field sensitivity may be further improved to surpass $\eta=2 \mu \mathrm{T} /(0.65 \mu \mathrm{m})^{2} / \sqrt{\mathrm{Hz}}$ through an increase in ESR contrast and a decrease in ESR linewidth by systematically optimizing the microwave power and laser power.

\section{Conclusions}

We have demonstrated that the total measurement time to obtain a magnetic field image at a comparable sensitivity is shorter in the wide-field magnetic field imaging than in the scanning probe method owing to the parallel acquisition of magnetic field images by an sCMOS camera. We expect that our wide-field magnetic field microscope will have a wide variety of applications, for example, in biological imaging and in characterizing solid-state devices and materials.

\section{Acknowledgments}

This work was partly supported by Grants-in Aid for Scientific Research on Innovative Areas "Topological Materials Science" (Nos. 15H05853, and 16H00978) and Grants-in-Aid for Scientific Research (Nos. 15H02117, and 15H03673) from the Japan Society for the Promotion of Science. 


\section{References}

1) C. L. Degen, Appl. Phys. Lett. 92, 243111 (2008).

2) G. Balasubramanian, I. Y. Chan, R. Kolesov, M. A.-Hmoud, J. Tisler, C. Shin, C. Kim, A. Wojcik, P. R. Hemmer, A. Krueger, T. Hanke, A. Leitenstorfer, R. Bratschitsch, F, Jelezko, and J. Wrachtrup, Nature 455, 648 (2008).

3) J.M. Taylor, P. Cappellaro, L. Childress, L. Jiang, D. Budker, P. R. Hemmer, A. Yacoby, R. Walsworth, and M. D. Lukin, Nat. Phys. 4, 810 (2008).

4) R. Schirhagl, K. Chang, M. Lorentz, and C. L. Degen, Annu. Rev. Phys. Chem. 65, 83 (2014).

5) L. Rondin, J.-P. Tetienne, T. Hingant, J.-F. Roch, P. Maletinsky, and V. Jacques, Rep. Prog. Phys. 77, 056503 (2014).

6) C. Granata and A. Vettoliere, Phys. Rep. 614, 1 (2016).

7) K. Hasselbach, D. Mailly, and J. R. Kirtley, J. Appl. Phys. 91, 4432 (2002).

8) N. C. Koshnick, M. E. Huber, J. A. Bert, C. W. Hicks, J. Large, H. Edwards, and K. A. Moler, Appl. Phys. Lett. 93243101 (2008).

9) D. Vsyukov, Y. Anahory, L. Embon, D. Halbertal, J. Cuppens, L. Neeman, A. Finkler, Y. Segev, Y. Myasoedov, M. L. Rappaport, M. E. Huber, and E. Zeldov, Nat. Nano. Technol. 9, 639 (2013).

10) D. C. Cox, J. C. Gallop, and L. Hao, Nanofabrication 1, 53 (2014).

11) E. M. Spanton, K. C. Nowack, L. Du, G. Sullivan, R.-R. Du, and K. A. Moler, Phys. Rev. Lett. 113, 026804 (2014).

12) Y. Shibata, S. Nomura, H. Kashiwaya, S. Kashiwaya, R. Ishiguro, and H. Takayanagi, Sci. Rep. 5, 15097 (2015).

13) A. M. Chang, H. D. Hallen, L. Harriott, H. F. Hess, H. L. Kao, J. Kwo, R. E. Miller, R. Wolfe, J. van der Ziel, and T. Y. Chang, Appl. Phys. Lett. 61, 1974 (1992).

14) A. Oral, S. J. Bending, and M. Henini, Appl. Phys. Lett. 69, 1324 (1996).

15) A. Sandhu, A. Okamoto, I. Shibasaki, and A. Oral, Microelectron. Eng. 73-74, 524 (2004).

16) A. Sandhu, K. Kurosawa, M. Dede, and A. Oral, Jpn. J. Appl. Phys. 43, 777 (2004).

17) Y. Martin and H. K. Wickramasinghe, Appl. Phys. Lett. 50, 1455 (1987).

18) D. Rugar, H. J. Mamin, P. Guethner, S. E. Lambert, J. E. Stern, I. McFadyen, and T. Yogi, J. Appl. Phys. 68, 1169 (1990).

19) S. McVitie, R. Ferrier, J. Scott, G. White, and A. Gallagher, J. Appl. Phys. 89, 3656 
(2001).

20) S. Steinert, F. Dolde, P. Neumann, A. Aird, B. Naydenov, G. Balasubramanian, F. Jelezko, and J. Wrachtrup, Rev. Sci. Instrum. 81, 043705 (2010).

21) L. M. Pham, D. Le Sage, P. L. Stanwix, T. K. Yeung, D. Glenn, A Trifonov, P. Cappellaro, P. R. Hemmer, M. D. Lukin, H Park, A. Yacoby, and R. L. Walsworth, New J. Phys. 13, 045021 (2011).

22) D.L. Sage, K. Arai, D. R. Glenn, S. J. DeVience, L. M. Pham, L. Rahn-Lee, M. D. Lukin, A. Yacoby, A. Komeili, and R. L. Walswort, Nature 496, 486 (2013).

23) D. A. Simpson, J.-P. Tetienne, J. M. McCoey, K. Ganesan, L. T. Hall, S. Petrou, R. E. Scholten, and L. C. L. Hollenberg, Sci. Rep. 6, 22797 (2016).

24) R.S. Schoenfeld and W. Harneit, Phys. Rev. Lett. 106, 030802 (2011).

25) Y. Miura, S. Kashiwaya, and S. Nomura, Ext. Abstr. Int. Conf. Solid State Devices and Materials, 2016, p. 837.

26) J. R. Rabeau, P. Reichart, G. Tamanyan, D. N. Jamieson, S. Prawer, F. Jelezko, T. Gaebel, I. Popa, M. Domhan and J. Wrachtrup, Appl. Phys. Lett. 88, 023113 (2006).

27) S. Pezzagna, B. Naydenov, F. Jelezko, J. Wrachtrup, and J. Meijer, New J. Phys, 12, 065017 (2010).

28) B. K. Ofori-Okai, S. Pezzagna, K. Chang, M. Loretz, R. Schirhagl, Y. Tao, B. A. Moores, K. Groot-Berning, J. Meijer, and C. L. Degen, Phys. Rev. B 86, 081406 (2012).

29) M. W. Doherty, N. B. Manson, P. Delaney, F. Jelezko, J. Wrachtrup, L. C. L. Hollenberg, Phys. Rep. 528, 1 (2013).

30) B.J. Maertz, A. P. Wijnheijmer, G. D. Fuchs, M. E. Nowakowski, and D. D. Awschalom, Appl. Phys. Lett. 96, 092504 (2010).

31) S. Felton, A. M. Edmonds, M. E. Newton, P. M. Martineau, D. Fisher, D. J. Twitchen, and J. M. Baker, Phys. Rev. B 79, 075203 (2009).

32) A. Dreau, M. Lesik, L. Rondin, P. Spinicelli, O. Arcizet, J.-F. Roch, and V. Jacques, Phys. Rev. B 84, 195204 (2011). 


\section{Figure Captions}

Fig. 1. (Color online) Schematic diagram of a setup for wide-field magnetic field imaging using an sCMOS camera.

Fig. 2. (Color online) Normalized photoluminescence intensity of NV ensembles in diamond at room temperature as a function of microwave frequency for the external magnetic fields (a) B // [001] and (c) B // [111]. (b, d) Schematics of magnetic field direction with respect to the crystal axis.

Fig. 3. (Color online) (a) Normalized photoluminescence intensity change by frequency modulation as a function of microwave frequency for the external magnetic field $\mathbf{B} / /$ [111]. (b) Magnified plot of (a) for microwave frequency between 2.7 and $2.8 \mathrm{GHz}$. 


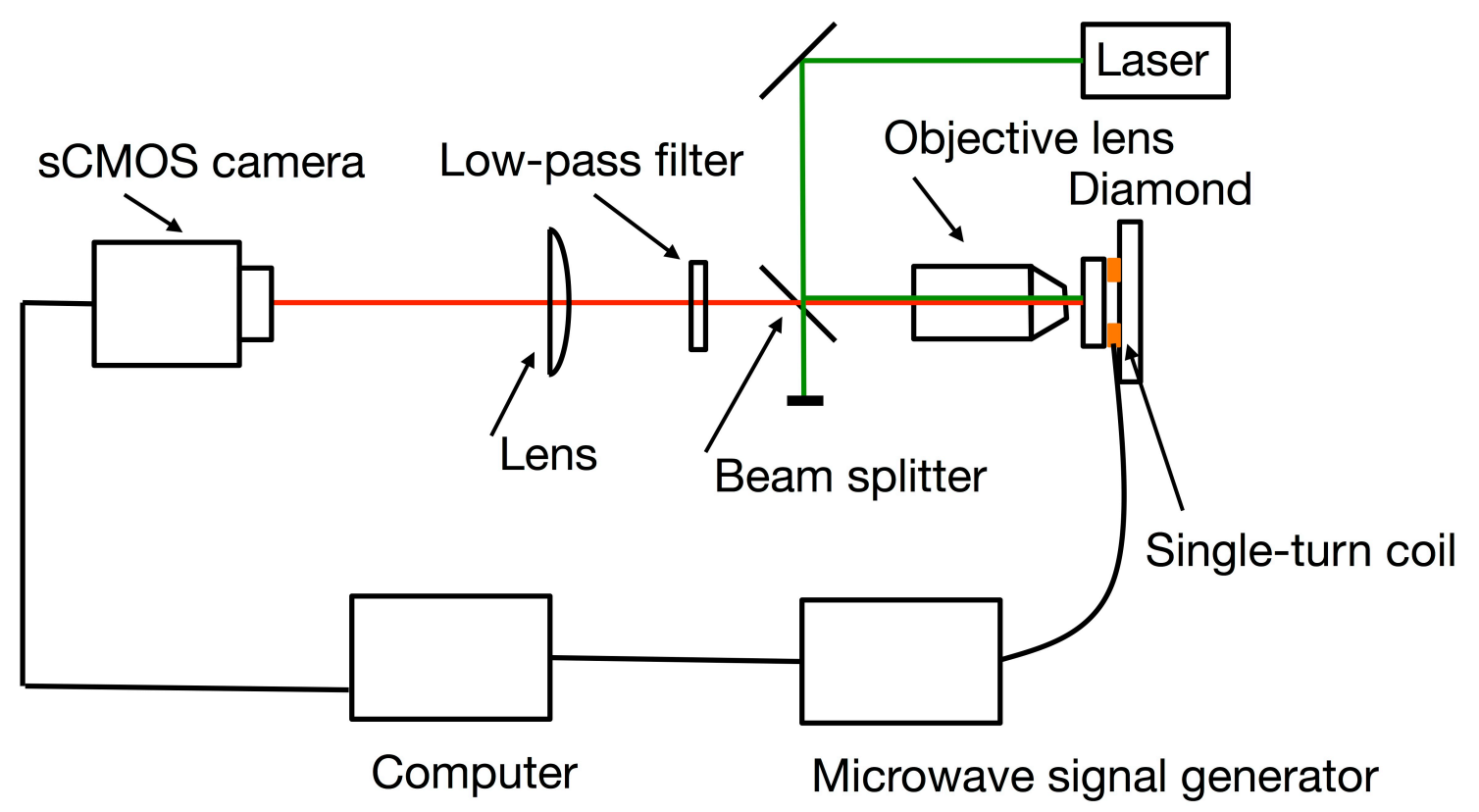

Fig. 1. (Color Online) 
(a)

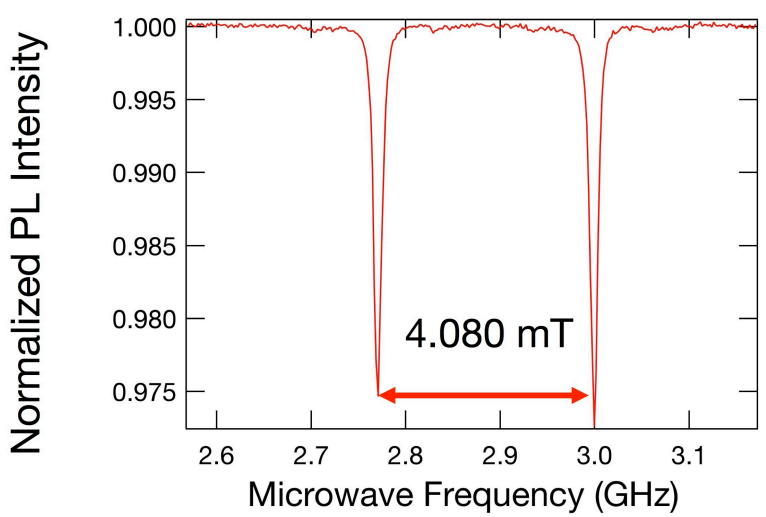

(c)

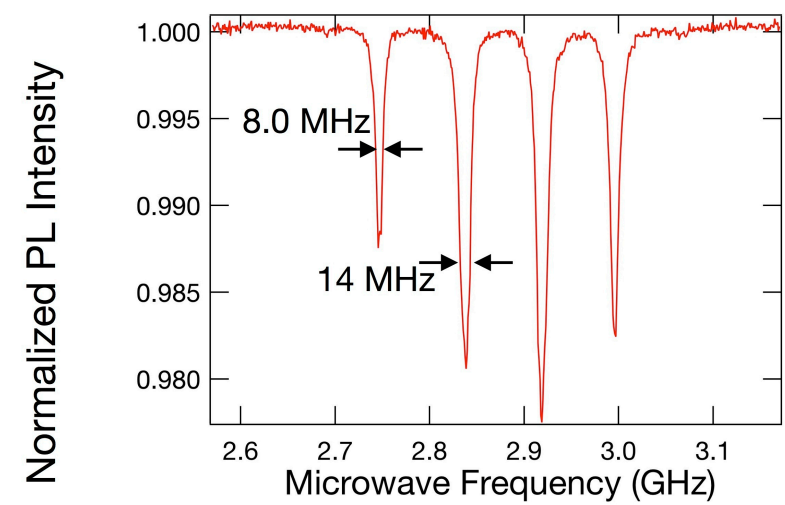

(b)

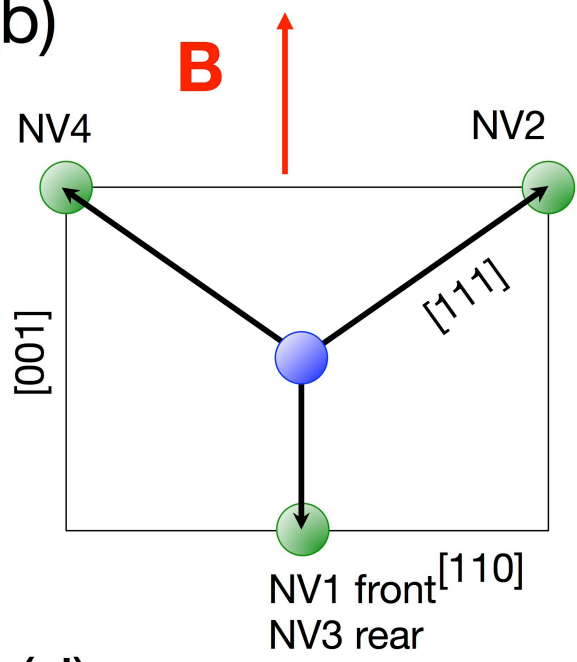

(d)

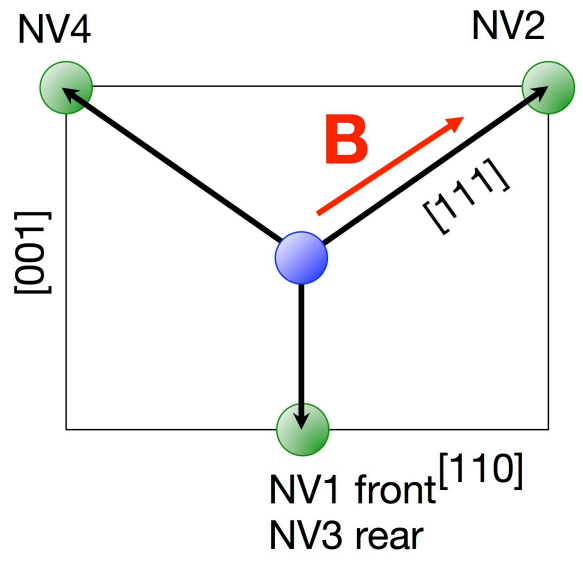

Fig. 2. (Color Online) 
(a)
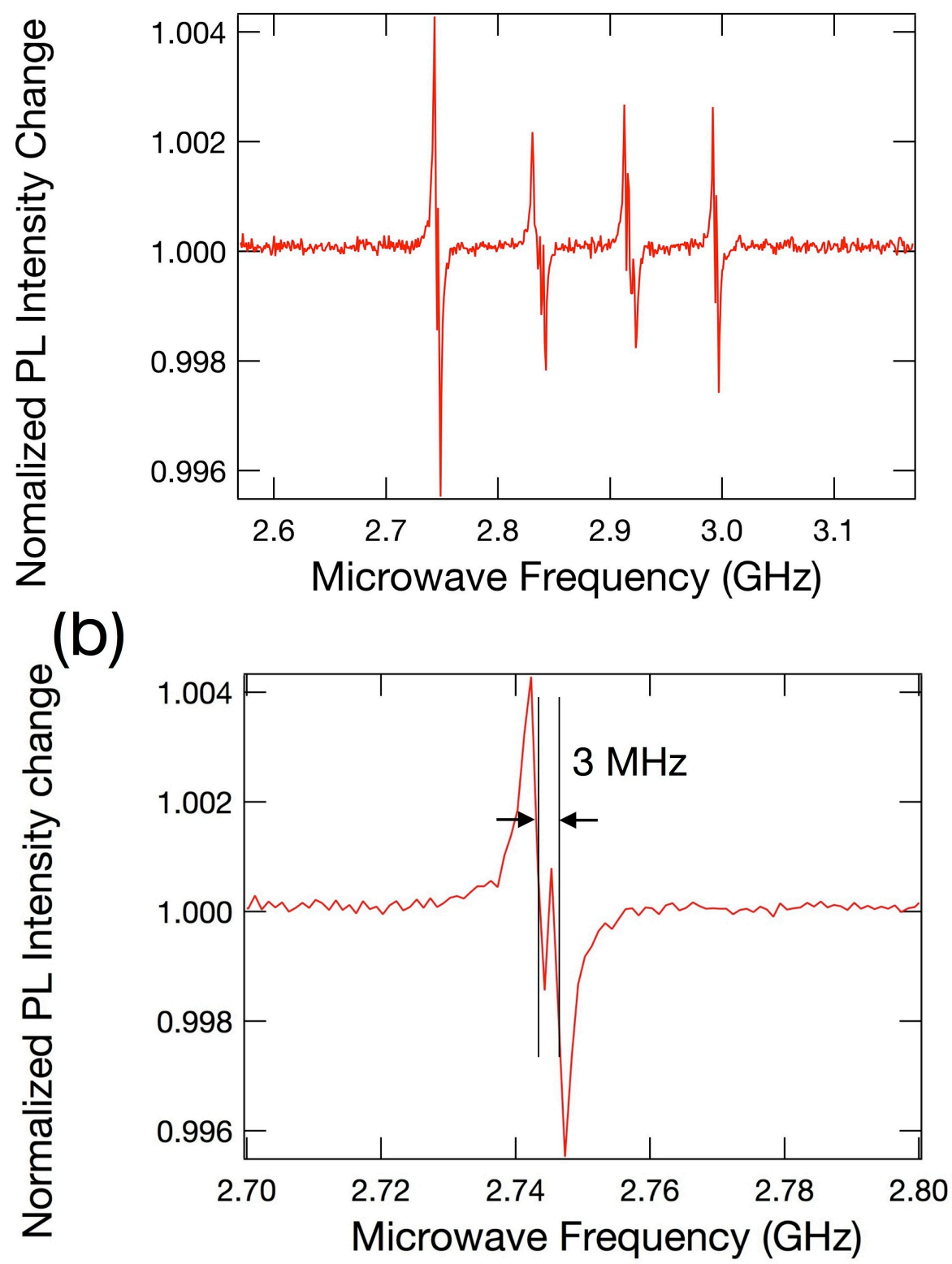

Fig. 3. (Color Online) 